\section{Improving diagnostic accuracy of endoscopic ultrasound}

\author{
"Wet suction technique": A novel way \\ to enhance the quality of endoscopic \\ ultrasound-fine-needle aspiration \\ aspirate. Results of a prospective, \\ single-blind, randomized, controlled trial \\ using a 22-gauge needle for endoscopic \\ ultrasound-fine-needle aspiration of \\ solid lesions
}

Rajeev Attam, Mustafa A. Arain, Stephen J. Bloechl, Guru Trikudanathan, Satish Munigala, Yan Bakman, Maharaj Singh, Timothy Wallace, Joseph B. Henderson, Marc F. Catalano, Nalini M. Guda

Gastrointestinal Endoscopy early online 2015

Endoscopic ultrasound (EUS) has revolutionized the practice of gastrointestinal endoscopy by making the endoscopic procedures safer and also venture into difficult endoscopic procedures. Because of its ability to provide high-resolution images, it has improved our diagnostic accuracy especially for submucosal lesions as well as pancreaticobiliary disorders. ${ }^{[1-3]}$ The addition of fine-needle aspiration (FNA) sampling to EUS has further improved its diagnostic accuracy. After passing the needle through the accessory channel of the echoendoscope, the FNA needle is passed into the lesion under EUS guidance. Currently, we use multiple types of needle and varying aspiration techniques without a clear consensus on the best sampling technique. The authors in the current study describe a novel technique of flushing the needle with $5 \mathrm{~mL}$ of saline solution to replace the column of air with saline solution before FNA with an aim of improving the quality of aspirates.

The authors conducted a prospective, two-center, randomized, single-blind, controlled study in 95 patients (117 lesions sampled) comparing the quality of aspirates obtained by using the wet suction technique (WEST) with those obtained by the conventional FNA technique (CFNAT) by using 22-gauge needles for EUS-guided FNA of solid lesions. The needle used for FNA was a 22-gauge needle (EchoTip Ultra HD; Cook Endoscopy, Winston-Salem, NC) with an identical suction method.

\section{Technique}

In the WEST, the needle was flushed with $5 \mathrm{~mL}$ of saline solution to replace the column of air and thereafter, a $10-\mathrm{mL}$ suction syringe, loaded to maximal suction was attached in a "locked" position to the needle. In the CFNAT, after removing the stylet, a $10-\mathrm{mL}$ syringe, loaded to maximal suction was attached in a "locked" position to the needle. In both the techniques, the suction was applied after the lesion was punctured. The procedures were performed using a linear Olympus echoendoscope (GF UCT 180; Olympus America Inc., Center Valley, Pennsylvania) with a Pro Sound processor (Aloka, Wallingford, Conn) under conscious sedation or monitored anesthesia care. Once inside the lesion, the needle was moved back and forth within the lesion 16-20 times to obtain an aspirate. During this step, the drops of saline could be seen moving into the suction syringe in the WEST method. The aspirate was delivered onto a slide using a stylet and thereafter the needles were flushed with $2 \mathrm{~mL}$ of saline to ensure removal of all aspirates into the formalin container. All solid lesions were sampled by using both the techniques in alternating manner with the same needle. In one of the centers (University of Minnesota Medical Center; UMN) an onsite cytopathologist was available and this determined the number of passes whereas at the second center (Aurora St. Luke's Medical Center; AMC) onsite cytopathologist was unavailable and, therefore, the number of passes was determined by the endosonographer (usually 4-6 passes per lesion).

All FNA specimens were reviewed and graded by two experienced cytopathologists ( 1 at each center) who knew clinical information pertaining to the case including age, sex, and site of lesion. Cellularity was assessed by using a 4-point scale $(0=$ no cells, $1=$ sparsely cellular, $2=$ moderately cellular, $3=$ highly cellular). Blood contamination was graded on a 3 -point scale $(0=$ free of blood, $1=$ contaminated with red blood cells, 2 = blood clots present). The specimen adequacy was graded on a 2-point scale (score 0 if the cytopathologist was unable to make a diagnosis and a score of 1 cytopathologist could make a diagnosis).

The most commonly sampled lesion was pancreatic followed by lymph nodes. Totally, 72 lesions were sampled at UMN with a mean number of passes per lesion being $4.20 \pm 1.31$ and at AMC, 45 lesions were sampled with a mean number of passes being $3.22 \pm 1.05$. A total of 63 lesions had a positive cytological diagnosis of malignancy (confirmed and/or

This is an open access article distributed under the terms of the Creative Commons Attribution-NonCommercial-ShareAlike 3.0 License, which allows others to remix, tweak, and build upon the work non-commercially, as long as the author is credited and the new creations are licensed under the identical terms.

For reprints contact: reprints@medknow.com 
suspicious for malignancy) whereas atypical cells were seen in three patients and in five patients, aspirates were nondiagnostic (pancreas, 1; lymph node, 4). Thirty-seven lesions were negative for malignancy, and nine other lesions were benign including a small GI stromal tumor. WEST was used as the first-pass technique in sampling 59 lesions (50\%), and the total number of passes using the WEST and CFNAT were 221 and 227, respectively.

In 112 of 117 sampled lesions, cytopathologists had adequate specimens to reach a diagnosis and of the five inadequate specimens, one was from a peripancreatic lesion and four from lymph nodes. A WEST cell-block had a significantly better specimen adequacy of 100 of 117 (85.5\%) compared with the CFNAT $(88 / 117,75.2 \% ; P=0.035)$. In seven lesions, the WEST aspirate was diagnostic for malignancy, whereas the CFNAT aspirates were either hypocellular or negative for malignancy. In one patient, CFNAT aspirate was diagnostic for malignancy, whereas the WEST aspirate was hypocellular. The WEST aspirate had a higher mean cellularity score of 1.82 compared with 1.45 with the CFNAT $(P=0.0003)$ with a larger proportion of aspirates in the WEST group being of moderate and high cellularity. The WEST provided higher mean cellularity scores even when it was not used as the firstpass technique ( 1.85 vs. $1.52 ; P=0.02)$ and when it was used as the first-pass technique, the difference was more pronounced (WEST: 1.79 , CFNAT: $1.38 ; P=0.0046$ ). There was no difference in the amount of blood contamination between the two techniques and also there were no adverse events related to FNA by either technique. The authors concluded that the novel WEST technique of EUS FNA resulted in significantly better cellularity and specimen adequacy in cell blocks of EUS-guided FNA aspirate of solid lesions than the CFNAT.

\section{Commentary}

The addition of FNA sampling to EUS has further improved its diagnostic accuracy and now EUS-FNA is the modality of choice for obtaining tissue for diagnosing various intraabdominal and mediastinal lesions. However, false negative rates as high as $19 \%$ is an important limitation of this technique, and poor cellularity of the aspirates is a common cause for this false negativity. ${ }^{[4,5]}$ Various modifications in the needle design, as well as in the technique like using reverse bevel-tip needles, and use of variable amounts of suction including fine-needle capillary sampling, in which the needle is passed into the target lesion and a specimen is obtained without the use of suction have been tested for improving the cytological yield of EUS FNA with varying results. ${ }^{[6]}$ The authors of the current study used this WEST technique after they hypothesized that the presence of a saline solution column might keep the needle from getting clogged while avoiding the inherent inconvenience of a metal stylet. The exact mechanism for improved results with the WEST technique are not known but the authors believe that a column of saline solution may allow for better transmission of applied suction compared with a column of air within the needle and saline solution may coat the inner lining of the needle thereby changing its surface properties and this possibly might lead to easier movement of aspirated into the needle. Moreover, similar blood contamination between the two techniques made authors hypothesize that the presence of saline solution could eliminate the "empty space" in the needle lumen for the tissue to bleed into. It is indeed a thought provoking study with interesting results that need to be replicated by other studies from other centers also, before the WEST technique of FNA becomes the standard of care. Furthermore, cytopathological grading by only one cytopathologist and availability of on-site cytological evaluation at only one of the two participating centers are important limitations of the study. It would also be interesting to find out whether using alternative solutions like heparin or heparinized saline could yield better results!.

Surinder Singh Rana

Department of Gastroenterology, Post Graduate Institute of Medical Education and Research, Chandigarh, India

Address for correspondence: Dr. Surinder Singh Rana Department of Gastroenterology, Post Graduate Institute of Medical Education and Research, Chandigarh - 160 012, India.

E-mail: drsurinderrana@yahoo.co.in

\section{References}

1. Rana SS, Vilmann P. Endoscopic ultrasound features of chronic pancreatitis: A pictorial review. Endosc Ultrasound 2015;4:10-4.

2. Rana SS, Sharma R, Sharma V, Bhasin DK. An unusual cause of gastric submucosal bulge on endoscopy. Endosc Ultrasound 2014;3:198-9.

3. Rana SS, Gonen C, Vilmann P. Endoscopic ultrasound and pancreas divisum. JOP 2012;13:252-7.

4. Dumonceau JM, Koessler T, van Hooft JE, Fockens P. Endoscopic ultrasonography-guided fine needle aspiration: Relatively low sensitivity in the endosonographer population. World J Gastroenterol 2012;18:235763.

5. Woolf KM, Liang H, Sletten ZJ, Russell DK, Bonfiglio TA, Zhou Z. False-negative rate of endoscopic ultrasound-guided fine-needle aspiration for pancreatic solid and cystic lesions with matched surgical resections as the gold standard: One institution's experience. Cancer Cytopathol 2013;121:449-58.

6. Wallace MB, Kennedy T, Durkalski V, Eloubeidi MA, Etamad R, Matsuda K, et al. Randomized controlled trial of EUS-guided fine needle aspiration techniques for the detection of malignant lymphadenopathy. Gastrointest Endosc 2001;54:441-7. 